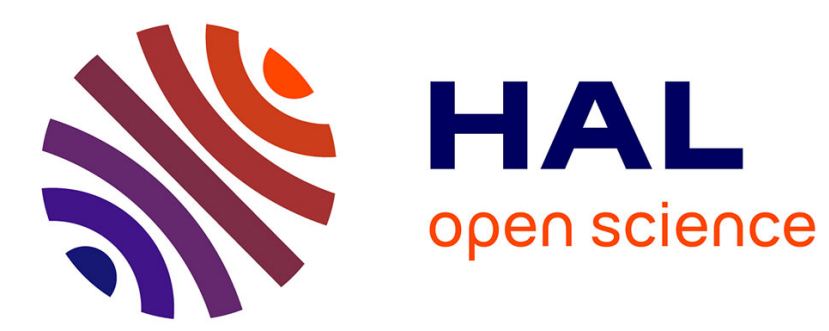

\title{
La séduction au service de la perfidie: représentation des missionnaires de la contre-réforme en Angleterre
}

\author{
Laurence Lux-Sterritt
}

\section{To cite this version:}

Laurence Lux-Sterritt. La séduction au service de la perfidie: représentation des missionnaires de la contre-réforme en Angleterre. XVII-XVIII Revue de la Société d'études anglo-américaines des XVIIe et XVIIIe siècles, 2008, Les formes de la séduction, 65, pp.57-75. 10.3406/xvii.2008.2370 . hal-01184572

\section{HAL Id: hal-01184572 \\ https://hal.science/hal-01184572}

Submitted on 17 Aug 2015

HAL is a multi-disciplinary open access archive for the deposit and dissemination of scientific research documents, whether they are published or not. The documents may come from teaching and research institutions in France or abroad, or from public or private research centers.
L'archive ouverte pluridisciplinaire HAL, est destinée au dépôt et à la diffusion de documents scientifiques de niveau recherche, publiés ou non, émanant des établissements d'enseignement et de recherche français ou étrangers, des laboratoires publics ou privés. 


\section{LA SÉDUCTION AU SERVICE DE LA PERFIDIE: REPRÉSENTATION DES MISSIONNAIRES DE LA CONTRE-RÉFORME EN ANGLETERRE.}

L'Angleterre de l'ère moderne construisit en partie son identité sur l'exclusion systématique de ses sujets catholiques. Dans des publications volontiers alarmistes, la propagande anti-catholique dénonçait le rôle dévolu aux femmes dans le projet subversif de la mission catholique. C'est en circonvenant les femmes, célibataires, veuves, épouses ou mères, que les papistes menaçaient l'intégrité de la nation. La controverse anti-catholique dénonce la stratégie de séduction du missionnaire catholique qui, tel le serpent avec Ève, s'en prend à la femme pour mieux détourner l'époux, et de proche en proche, renverser les valeurs du royaume.

The construction of early modern English identity relied partly upon the systematic ostracizing of "papist 》 subjects, who came to embody the very opposite of Englishness. In a plethora of alarmist publications, polemicists insisted upon the importance of women in the subversive project of the Catholic mission: it was by corrupting spinsters, widows, but also housewives and mothers that papists jeopardized the integrity of the nation. The antiCatholic controversy therefore exposed the seductive strategies of priests in order to unmask their duplicity and unveil their truly evil nature: like the serpent who tempted Eve to defy God, missionaries would seduce women as an indirect means to ensnare their husbands, influence their families, and eventually overthrow the values of the kingdom.

Alors que l'Angleterre tente, durant toute l'époque moderne, de définir son identité religieuse et de se montrer comme une nation unie autour d'une Anglicana Ecclesia, séparée de Rome depuis Henri VIII, ${ }^{1}$

1. En 1533, le souverain reniait l'autorité du pape : «[...] this realm of England is an Empire [...] governed by one supreme Head and King » (Act in Restraint of Appeals to Rome). L'année suivante, l'acte de Suprématie officialisait la naissance de l'Église d'Angleterre, sous l'autorité suprême du roi, qui devenait ainsi chef de l'Église aussi bien que de l'État: « [...] Be it enacted by authority of this present 
l'existence d'une communauté catholique au sein du royaume, si minoritaire et clandestine soit-elle, ne manque pas de poser de graves difficultés. Car, depuis le règne d'Élisabeth I (1558-1603), ces sujets anglais, dont l'allégeance est partagée puisqu'ils obéissent au pape autant qu'au monarque, deviennent l'objet de peurs et de soupçons qui s'expriment dans l'abondant corpus des pamphlets anticatholiques. On se défie d'eux et l'usage du terme méprisant de «papiste», montre la cassure grandissante entre les loyaux sujets de la couronne et ceux que l'on représente comme des traitres potentiels, alliés aux entreprises de Rome pour détruire le royaume. Cette peur, culmine en 1688 avec la Glorieuse Révolution et l'exclusion du roi Jacques II (1685-88).

Les crises qui jalonnent la période exaspèrent la dichotomie entre protestants et catholiques; Élisabeth I ne fut-elle pas menacée par diverses conspirations ? ${ }^{2}$ Jacques I (1603-25) ne fut-il pas victime du complot des Poudres, le 5 novembre 1605? Et Charles II (1660-85), ayant restauré la monarchie et l'Église anglicane après la République puritaine d'Oliver Cromwell, ne fut-il pas l'objet des machinations de Titus Oates en 1678 ? Selon les polémistes, ces attentats sont la preuve de la duplicité des «papistes». Durant des décennies, foisonnent les titres qui cherchent à alarmer le public en s'attardant sur la duplicité du catholique, qui devient par définition l'ennemi du royaume souverain d'Angleterre. Les études d'historiens comme Michael Questier, Peter Lake et Alexandra Walsham, ainsi que celles de critiques littéraires comme Frances Dolan, Arthur Marotti, Alison Shell ou Raymond Tumbleson tentent aujourd'hui de distinguer le réel de l'imaginaire dans cette polémique anticatholique.

Parliament that the King our sovereign lord, his heirs and successors kings of this realm, shall be taken, accepted and reputed the only supreme head in earth of the Church of England called Anglicana Ecclesia [...]».

2. Entre 1569 et 1586, le règne d'Élisabeth I est menacé par une succession de complots visant à l'assassiner et à la remplacer par sa cousine, la catholique Marie Stuart, reine d'Écosse. En 1569, on découvre l'insurrection des contes de Westmoreland et de Northumberland; puis c'est le banquier florentin Roberto Ridolfi qui ourdit un complot en 1570. En 1583, le catholique Sir Francis Throckmorton, en contact fréquent avec Marie Stuart, est exécuté pour haute trahison. Enfin, en 1586, Sir Anthony Babington est à son tour condamné pour des faits similaires; les preuves accumulées contre Marie Stuart convainquent Elisabeth de signer l'arrêt de mort de sa cousine. Le contexte international, la guerre contre l'Espagne (et notamment l'épisode de l'Armada en 1588) contribuent également à attiser les sentiments anticatholiques. 
Le propos de la présente étude est un peu différent: il ne s'agit pas ici d'estimer la véracité des accusations formulées, mais d'étudier le discours et la rhétorique mis en œuvre pour diaboliser le catholique dans l'imagination anglaise. On remarque en effet que, si l'agitateur politique fait couler beaucoup d'encre, il inquiète moins que son confrère le missionnaire qui, loin de la cour, œuvre en secret dans les familles du royaume. Ces prêtres, condamnés à la clandestinité par la sévérité des lois pénales qui les rendent passibles de la peine de mort, prennent une dimension sans précédent dans l'imaginaire collectif de l'époque moderne. Les plus grands auteurs, même s'ils restent en marge de la diatribe anticatholique, sèment leurs ouvrages de commentaires qui témoignent du climat de peur grandissant. Ainsi John Milton, dans Areopagitica, dénonce les "papistes» comme des imposteurs au service de l'oppression romaine, "falsest seducers and oppressors of men » (245). Jour après jour, indétectables, ils travaillent à la conversion de l'Angleterre et leur discrétion tourmente notamment les auteurs anticatholiques qui, pour servir leur propos, choisissent de mettre l'accent plus que de coutume sur le rôle des femmes.

Alexandra Walsham a démontré que la surreprésentation féminine dans la communauté récusante, dont attestent les recensements officiels, étaient souvent le résultat de pactes domestiques passés entre époux afin d'éviter les sanctions pénales. Or, ce cas de figure n'est pas mis en avant par les polémistes du temps qui dénoncent au contraire une stratégie visant à multiplier les conversions féminines. L'anticatholicisme anglais contribue donc à donner aux femmes une place centrale dans la représentation du danger "papiste»: peintes en victimes d'abord innocentes puis consentantes, elles sont un élément-clé du discours de propagande protestante visant à diaboliser les missionnaires, et les jésuites en particulier. C'est, nous dit-on, en séduisant les femmes que le prêtre s'immisce dans la vie privée des familles, petites et grandes, pour y multiplier ses œuvres subversives (Dolan 53). On argue que l'entreprise de séduction est aisée, car l'intellect féminin n'offre guère de résistance: ainsi manipulées par les missionnaires, riches veuves ou célibataires fortunées sont pour eux d'idéals mécènes qui, protégées par l'égard dû à leur statut social et à leur sexe, offrent la sécurité et le financement sans lesquels la Mission ne saurait remplir sa fonction. Mais les missionnaires ne séduisent pas seulement pour s'approprier des ressources. Ils cherchent à attaquer le tissu social, et prennent pour cibles les mères de famille, même les plus humbles, car elles permettent la conversion des époux et des enfants (Yates 64); 
cette stratégie leur permet enfin d'usurper l'autorité du paterfamilias, de renverser les rôles domestiques, et finalement de détruire l'ordre établi, mettant en péril les valeurs fondamentales du royaume.

Dans A Declaration of Egregious Popish Impostures, Samuel Harsnett présente les jésuites comme d'habiles séducteurs. Il s'agit de détourner les innocents du droit de chemin, de les convaincre par des procédés souvent malhonnêtes. Selon lui, le catholicisme, obscurantiste par nature, mêle tradition, idolâtrie et superstition; c'est une supercherie mise au point pour duper les esprits les plus vulnérables par des procédés rhétoriques et scéniques destinés à impressionner et à influencer le public. Harsnett se penche sur certains cas de possession démoniaque et sur les exorcismes qui les accompagnent: ce sont là, écrit-il, de pures mises en scène où acteurs et actrices ont appris et répété leur rôle. Il recueille le témoignage d'une certaine Anne Smith, prétendue possédée repentie, qui reconnaît n'avoir fait que se conformer à un scénario : «she learned her cue, to come into her fits » (191). Le polémiste s'appuie plus loin sur l'examen de Sara Williams pour dénoncer l'impudeur des officiants, qui, écrit-il, ne reculent devant rien pour choquer l'auditoire et lui faire accroire les pires mensonges : en partant des pieds, les prêtres posent leurs mains sur tout le corps de la jeune femme en remontant vers la tête, pour y repousser le démon qui sera alors expulsé par la bouche, les oreilles et le nez. Grâce aux pouvoirs de reliques sacrées, ils affirment avoir un jour expulsé un démon des parties génitales de la possédée (202). Selon Harsnett, l'exorcisme n'est qu'un stratagème qui doit d'être si répandu à sa faculté d'impressionner les foules, produisant à coup sûr l'effet désiré. Dans son ouvrage, le pamphlétaire exhorte ses compatriotes à ne pas se laisser ainsi mystifier: "To the Seduced Catholics of England : Seduced and disunited brethren, there be two grand witches in the world, that seduce the souls of the simple and lead them to perdition: lying wonders and counterfeit zeal». L'ancienne religion, écrit-il, abuse de la crédulité du peuple: «[Catholicism is] the only religion to catch fools, children and women, by reason it is naught else, save a conceit pageant of puppets » (A2 et 20).

Si les prêtres sont représentés comme autant de séducteurs visant à impressionner les esprits faibles, la polémique avance donc que les femmes, par la vulnérabilité intrinsèque à leur sexe, sont des proies toutes désignées. John Gee enquête sur les méthodes mises en œuvre par les «grands maîtres de la séduction, particulièrement les jésuites» 
(New Shreds B) et dénonce leur attitude prédatrice: «[they] steal away the hearts of the weaker sort, and secretly [...] creep into houses, leading captive simple women loaden with sins and led away with diverse lusts » (The Foot out of the Snare 3). Grâce à l'analyse de plusieurs cas particuliers, il s'efforce de percer à jour les pratiques employées pour convertir les jeunes femmes. Il évoque d'abord une certaine Mary Boucher qui, en service chez une dame catholique, est convertie par trois jésuites, Fisher, Wiseman and Ireland, qui la convainquent de renoncer à la religion protestante et de devenir religieuse. L'accent est mis sur les artifices de théâtre destinés à terrifier la victime: tandis que les clercs évoquent les tourments de l'enfer auxquels son hérésie la condamne, trois éclairs aveuglants déchirent la pénombre, suivis de l'arrivée spectaculaire d'un spectre féminin tout vêtu de blanc, dont les admonitions effraient tant la jeune femme qu'elle se convertit bientôt au catholicisme. Interrogée par l'auteur, Mary Boucher se dit victime d'un stratagème mêlant mise en scène dramatique et sorcellerie, tandis que Gee s'insurge contre la couardise des prêtres qui s'en prennent au sexe faible: «with what creeping sly stealth the Master gamesters the Jesuits do drive the female partridges into the net by the help of the setting dog of sneaking visions and phantasms » (New Shreds 23).

Les écrits protestants restent pourtant ambigus envers celles qu'ils décrivent comme les victimes des jésuites : elles sont en effet considérées à la fois comme des proies sans défense et des manipulatrices qui, une fois converties, se révèlent aussi redoutables que leurs confesseurs, jouant des préjugés culturels envers leur sexe pour mieux dissimuler leurs actions. Les polémistes prétendent qu'après avoir été séduite et trompée, la catholique devient à son tour séductrice et trompeuse; ils étaient leur thèse sur les récits des missionnaires eux-mêmes : il n'est pas rare en effet que, dans leurs écrits, les jésuites, rendant compte du progrès de leur mission, louent le travail de leurs associées et leur témoignent respect et gratitude. Par exemple, Henry Garnet (15551606), supérieur des jésuites en Angleterre, rend hommage au courage de sa partenaire en religion, Anne Vaux (1562-1637?), et de sa sœur ainée, la veuve Eleanor Brookesby (c.1560-1626), qui toutes deux accueillent les réunions annuelles de la Compagnie de Jésus dans leur demeure de Great Ashby, Leicestershire, jusqu'en 1600. Conscientes que la sécurité de la mission toute entière repose sur leurs épaules, elles savent exploiter l'opinion de leur époque qui ne voit en la femme qu'un être faible et inconstant. Lors d'une fouille en octobre 
1591, Anne Vaux manipule ainsi avec tact ces préjugés. Tandis que les cinq jésuites et les deux séminaristes présents dans la maison sont conduits dans les cachettes ménagées pour eux, et qu'Eleanor se réfugie dans une autre, Anne se prépare à accueillir les poursuivants et leurs hommes de main. Mais elle demande d'abord un moment de répit, prétextant, à l'heure matinale de la perquisition, ne pas être décemment habillée pour les recevoir. Garnet applaudit au sang-froid de sa pénitente, dont l'assurance laisse aux serviteurs le temps de faire disparaître toute trace de pratique catholique. Quand enfin elle ouvre la porte, c'est en parfaite maîtresse de maison qu'elle offre des collations aux perquisiteurs, permettant encore une fois à son personnel de maison de se rendre dans les pièces de l'arrière pour y dissimuler livres ou objets de culte, tandis que les poursuivants se réjouissent du bon accueil qui leur est fait.

En effet, les jésuites l'ont bien compris, ce n'est pas malgré, mais à cause de leur sexe que ces femmes sont utiles à la Mission, car il leur permet d'utiliser la misogynie établie de leur époque pour devenir des alliées irremplaçables. Garnet, organisateur pragmatique d'une mission clandestine dont il doit assurer la sécurité, salue l'aplomb d'Anne Vaux et se félicite d'avoir une associée si compétente. Mais de tels épisodes sont interprétés très différemment par ses détracteurs protestants : si les jésuites recherchent chez les femmes une protection qu'aucun homme n'aurait pu leur garantir, c'est que ce sont des imposteurs qui ne reculent devant rien pour exploiter ensuite celles dont ils ont gagné la confiance.

Le respect mutuel et l'entraide sur lesquels insistent les récits des missionnaires sont absents des publications protestantes, qui adoptent une perspective mieux faite pour échauffer le sentiment anticatholique. On remarque par exemple que les célibataires ont d'autres attraits que la seule protection qu'elles peuvent offrir. Les registres récusants montrent que les femmes fortunées, célibataires ou veuves, figurent en première place au rang des organisatrices et des piliers de la mission catholique en Angleterre. Les traités anticatholiques s'emploient à démontrer que cette incidence n'est autre que le fruit de la stratégie des missionnaires, qui cherchent à s'approprier logements et ressources. Gee montre que les prêtres s'enrichissent aux dépens de leurs alliées et dévoile les agissements des jésuites Fisher et Wiseman qui, en 1623, convoitaient les mille livres de rentes de l'orpheline Francis Peard. Usant du même artifice employé pour convertir Mary Boucher 
- le spectre féminin vêtu de blanc qu'annoncent d'éblouissants éclairs ils persuadent Francis Peard de prendre le voile, non sans l'avoir d'abord convaincue de mettre sa bourse au service de la Compagnie. Gee utilise encore l'image du prédateur et de sa proie: «they laboured to get this fat fish into their nett» (New Shreds 10), mais il souligne cette fois l'avidité des jésuites : «like two cunning gray-hounds that could well serve one another's turn for the catching the poor hare, with some other journey-men of their trade, [...] every one of them began to pluck a feather from the plume of her estate » (14).

De nombreux pamphlets, tels The Jesuites Intrigues de Henry Compton, dénoncent ces mêmes exactions : «[...] women, $[\ldots]$ being persuaded by these fathers to despise the World, are by Them, in requital made a Harvest of, being wheedled out of rich movables and other considerable matters »(7). Compton prétend reproduire les Instructions données par la Compagnie à ses missionnaires. Le chapitre 7, «How to keep Widows to our selves, so far as concerns the disposing of their Estates » montre que le jeu de la séduction, loin d'être une fin en soi, n'a d'autre but que de s'approprier les ressources des dames ainsi charmées :

Visit the Widows, as often as we may be welcome, entertaining them with pleasing discourses, and godly stories, and keep up the cheerfulness of their humour, and never be too severe with them in Confession, lest they take a distaste at us : unless there be no hopes left of making any advantage by them (38).

Compton, prétendant toujours à l'authenticité, montre que les jésuites ne reculent devant rien pour séduire de nobles veuves dont ils pourront dilapider la fortune; le chapitre des Instructions intitulé «How to procure the friendship of rich Widows» indiquerait, selon lui, la marche à suivre :

For this purpose must be called out some of the Fathers of the liveliest, fresh complexions, and of a middle age. These must frequent Their houses. [...] we must put a Confessor to them, that shall persuade them to continue in their widowhood [...]. Set them up a little Chapel, and an Altar neatly furnished, the minding of which may put the thoughts of a Husband out of their heads (35).

Les insinuations sensuelles sont à peine déguisées, et la séduction spirituelle s'allie au charme physique et personnel des jeunes prêtres en mission. 
Cette représentation, fruit d'une imagination protestante attisée par la peur et l'animosité, semble justifiée par l'interprétation faite des écrits des missionnaires. Comme son supérieur Garnet, le jésuite John Gerard (1564-1637) insiste souvent sur la dimension pragmatique de ses choix. Il avoue sans détours avoir incité sa bienfaitrice, Elizabeth Vaux (née Roper, d. 1627), à renoncer au couvent après la mort de son mari; en demeurant en Angleterre, elle permettait en effet à la mission de bénéficier de sa protection, de son réseau social, mais aussi de ses finances et de ses domaines. Garnet lui-même prodigue ces conseils à Anne Vaux, sa compagne en religion durant plus de vingt ans. Entre son arrestation le 27 janvier et son exécution le 3 mai 1606, il lui demande de continuer à servir en Angleterre et de ne pas entrer dans l'un des couvents du Continent, dont l'austérité ne pourrait que nuire à une santé déjà fragile (Caraman, Garnet 423). La sollicitude et la bienveillance du clerc à l'égard de sa pénitente se doublent de considérations pratiques, et si Garnet souhaite sincèrement épargner la santé d'Anne Vaux, la polémique protestante retiendra surtout qu'il cherche à exploiter son statut social et sa féminité pour protéger les activités de ses coreligionnaires en Angleterre.

Si les riches célibataires offrent ressources et protection, épouses et mères de famille représentent elles aussi d'idéales protectrices. Les explications de ce phénomène sont aussi variées que complexes, et continuent d'alimenter les études historiques. Godfrey Anstruther plaida d'abord pour une affinité quasi hormonale entre féminité et catholicisme, la sensibilité féminine étant particulièrement attirée par le mysticisme et les rituels de l'Église de Rome. Plus tard, John Bossy décrit le catholicisme anglais de l'époque moderne comme un «matriarcat»; il met en avant l'importance du système pénal qui, en forçant les catholiques à cacher leur foi, participa au repli de leurs pratiques religieuses sur la sphère domestique, domaine d'influence féminine par excellence. Plus récemment, Alexandra Walsham a imposé sa démonstration selon laquelle l'importance des femmes dans la communauté récusante pouvait s'expliquer, entre autres raisons, par l'inefficacité des lois anticatholiques (78-81): les sanctions pour récusance ${ }^{3}$ portent exclusivement atteinte à la richesse et à la propriété,

3. L'adjectif « papiste » est utilisé en règle générale par les protestants dénonçant tous les catholiques, qui par définition reconnaissent l'autorité papale en matière de religion. Au contraire, le terme "récusant » est un terme légal se rapportant à toute personne refusant de se présenter au service dominical anglican. Par glissement, le terme fut employé pour définir les catholiques refusant ouvertement de se conformer 
or la femme mariée, en tant que feme covert, ne possède aucun capital en son nom propre et ne peut donc être punie par la loi. En 1610, le parlement fait face à ce problème et vote une loi spécifique, «An Act for the Administration of the Oath of Allegiance and the Reformation of Married Women recusants »; l'épouse refusant de prêter le serment d'allégeance encourt une peine de prison, tandis que son mari est condamné à payer 10 livres par mois jusqu'à sa relaxe, ou, s'il ne le peut, à renoncer à un tiers de son patrimoine. C'est donc toujours le paterfamilias qui est sanctionné, puisque, selon la loi, c'est à lui qu'incombe la conformité religieuse de son foyer. Dans ce contexte, les épouses bénéficient d'une liberté religieuse dont leurs maris ne jouissent pas. Selon Walsham, bien des couples concluent alors un pacte domestique, l'époux s'en remettant à sa femme pour garantir la foi catholique de la famille; elle sera récusante tandis qu'il deviendra schismatique, sa conformité publique le protégeant des poursuites. L'État anglais, sans le vouloir, semble avoir contribué à donner aux femmes un rôle véritable dans l'organisation de la communauté catholique clandestine.

Mais cette explication suppose une réelle connivence entre époux; les polémistes de l'époque, eux, préfèrent souligner la malveillance des prêtres qui corrompent les liens sociaux en séduisant les mères de famille. Une fois encore, les pratiques des jésuites sont l'objet des lectures les plus sévères. John Gerard explique que c'est en raison de leur docilité naturelle que les femmes ne persistent pas dans l'hérésie de façon aussi opiniâtre que leurs époux; c'est pourquoi il préfère tenter de les convertir quand elles sont seules, comme dans le cas de Mary Mulshaw, qu'il instruit en l'absence de son mari, Sir Everard Digby. Puis, fort de son alliance avec l'épouse, et grâce à l'aide de son coreligionnaire Roger Lee, il entreprend la conversion du paterfamilias lui-même, qu'ils n'obtiendront qu' in extremis alors que celui-ci gît sur son lit de mort. Pour le jésuite, il s'agit de faire preuve de sagacité et d'utiliser tous les atouts pour s'assurer de l'heureuse issue de ce moment délicat, qui met en jeu le repos éternel de l'âme: la collusion avec Mary Mulshaw est nécessaire pour venir à bout de l'entêtement de Digby et lui assurer la bienveillance de Dieu. Il est impossible de

ou de prêter serment d'allégeance au monarque qui, en Angleterre, était reconnu comme autorité suprême pour l'Église comme pour l'état. Les récusants se différencient des schismatiques (dits "Church papists ») qui, souvent pour échapper aux sanctions, assistent à l'office et prêtent serment publiquement, bien qu'étant catholiques en privé. 
savoir si les auteurs protestants avaient pu lire les récits de mission de leurs ennemis «papistes», mais il ne fait aucun doute qu'ils en connaissaient la teneur. Or, pour eux, les mots de Gerard indiquent un calcul, avec la complicité de l'épouse, pour «capturer» Everard Digby: «[...] all three of us got together and discussed the best way of catching her husband in St Peter's net. [...] His illness gave me the opening I wanted» (Caraman, Gerard 165). La métaphore de la capture d'une proie, omniprésente dans les publications anticatholiques, se retrouve (avec une intention différente), sous la plume du missionnaire, et donne l'occasion à ses critiques de dépeindre les jésuites en agents du mal qui, tel le serpent qui tenta Ève pour atteindre Adam, séduisent les femmes afin de mieux corrompre les hommes: «seek they not to seduce poor simple women, that they may entice their husbands, as Eva did Adam? » (Baxter 37). Les jésuites sont représentés comme des empoisonneurs séduisant les femmes pour infecter ensuite les hommes et les enfants, car ce sont les mères qui amènent leur progéniture au catholicisme: «young imps that [...] have sucked poison from their mothers' breasts » (Baxter 178). L'influence des récusantes sur leurs enfants est en effet un fait reconnu qui amène le Parlement à étudier, entre 1605 et 1677 , de nombreuses propositions de loi, visant à les soustraire à l'influence jugée néfaste de leur mère. John Gerard lui-même admet utiliser la conversion d'une mère pour amener celle de toute la famille (Caraman, Gerard 189).

Si les polémistes anticatholiques se concentrent sur les récusantes, c'est parce que celles-ci jouent un rôle important dans ce qu'ils voient comme une stratégie permettant aux prêtres de s'immiscer dans la société anglaise pour la corrompre à l'insu des sujets protestants. Julian Yates remarque que les missionnaires sont perçus comme des parasites, qui infestent les domaines du privé autant que du public et mettent en danger toute la structure du royaume par leur travail de sape : "[t]he 'scandal' of recusancy was thus not so much the beliefs of English Catholics so much as the manner of their existence, the fact that they inhabited the fabric of the realm and did not resist openly » (172).

C'est, entre autres, l'identité catholique anglaise, multiple, variée et déroutante, qui exacerbe cette peur de l'ennemi domestique. En effet, si les récusants, qui refusent le service anglican, sont facilement identifiables, tous les «papistes» ne le sont pas. Certains, pour éviter les sanctions, sont d'occasionnels conformistes, qui assistent à l'office 
et prêtent serment d'allégeance au monarque. Ces «Church papists» sont souvent perçus comme les plus hypocrites, car ils sont difficiles à distinguer des Anglicans :

conformity with the Oath of allegiance and other outward formal satisfaction of the State, concurring with a resolution to continue in Popery, is far more pernicious to the State then open and professed Recusancy (Bernard B3).

Thomas Bell soutient que la dissimulation est inscrite dans le mode d'action des missionnaires :

I have with great watchings, painful studies, and nightly lucubrations found out the secret caves, dens and holes, to which the Romish fox that devours the innocent lambs of Christ [...] resorts usually and hides himself therein from time to time covertly 》 (Bell, Romish Foxe A4).

Plus tard, il développe encore son portrait du jésuite en utilisant cette fois l'image du caméléon: «[...] if you ask them what is a Jesuit, their answer is, every man. Implying, that they are creatures which vary their colours like the Cameleon, according to the object» (Popish Tyrannie 54); il qualifie enfin les prêtres de «parasites du pape »: «the Pope's parasites » (Downfall of Poperie 16), dont on ne connaît la présence que trop tard, alors que l'infrastructure de l'édifice est déjà affaiblie. Afin de le démasquer, Baxter suggère de placer sur la poitrine de chaque "papiste" une fenêtre à travers laquelle on pourrait entrevoir son âme et connaître ainsi, par transparence, la vraie nature de ses pensées et de ses convictions (Toile 110). Et l'auteur de se faire le porte-parole de l'obsession nationale envers les intentions secrètes des catholiques lorsqu'il conclut: «forbearance of household foes can be no good policy» (169). En 1614 , le parlement envisage même de contraindre les catholiques à porter toques et chausses de couleur jaune, couleur symbolisant la traitrise, afin de pouvoir les reconnaître d'un simple coup d'œil parmi ses voisins, ses associés, et même ses amis. Car c'est là l'un des aspects les plus dérangeants de la séduction catholique : l'ennemi, nous prévient-on, avance masqué et souvent sous les traits agréables et familiers d'un proche. Le lien marital lui-même est susceptible d'être souillé par ceux qui s'immiscent dans l'affection des femmes pour y supplanter leurs époux, corrompant ainsi un lien sacré.

Si les prêtres s'allient aux femmes pour bénéficier de leur protection et de leurs réseaux sociaux, de telles collaborations entre 
les sexes se prêtent volontiers à des interprétations d'ordre moral. Les clercs sont autorisés à pénétrer les espaces féminins les plus secrets, ceux dont sont normalement exclus les hommes; dans ce milieu caché, ils occupent les espaces les plus privés, les chapelles clandestines et les «priests' holes » dissimulés dans l'architecture même des demeures, mais aussi les boudoirs et les chambres à coucher. Comptant sur la bienséance des officiers pour ne pas fouiller la couche encore tiède d'une dame de bonne famille, ils n'hésitent pas à se réfugier dans le lit de leur bienfaitrice pour échapper aux poursuivants. À l'inverse, les récusantes passent de longues heures dans les quartiers des prêtres ou les ravitaillent dans l'espace confiné de leurs cachettes. Le débat anticatholique fait grand cas des «liaisons ambiguës » entre pénitentes et hommes d'Église, souvent présentées comme scandaleuses, et que Coleen Seguin qualifie de «cocufiage spirituel» («spiritual cuckoldry» 161).

Dans le débat anticatholique, la pénétration d'espaces sexués paraît symptomatique d'une moralité corrompue, et l'aspect spirituel de ce cocufiage cède le pas à des anecdotes salaces qui ne manquent pas de faire sensation. L'amitié qui, pendant vingt ans, lia Garnet à Anne Vaux donne lieu à des calomnies lors du procès de ce dernier. On remarque qu'Anne l'a accompagné en tout lieu et qu'ils ont vécu, dit-on, comme mari et femme. On en apporte comme preuve une lettre d'Anne Vaux à son confesseur emprisonné, signée A. G., pour Anne Garnet. Le juge, Lord Salisbury, se saisit de cette correspondance pour démontrer l'immoralité du jésuite: "What, you are married to Mrs. Vaux : she calls herself Garnet. What! Senex fornicarius! (174). Les protestations du prêtre n'y feront rien, sa relation avec sa partenaire de mission est à jamais entachée de suspicion. La séduction attribuée aux prêtres n'est donc pas exclusivement de nature morale, mais peut bel et bien prendre des dimensions physiques, voire sexuelles, que les polémistes se plaisent à dévoiler, dénonçant la prétendue chasteté des prêtres comme une simple ruse destinée à inspirer la confiance :

You abominable Whore-masters, you filthy fornicators, you stinking Sodomites, you deceitful Deflowerers of maids, you devilish defilers of men's wives, you cankered corrupters of widows, and you lecherous locusts, may lie with your whores and harlots all night, and the next day after go to Masse. (Becon 157-59)

L'activité missionnaire est associée à la débauche; on prétend même que les jésuites donnent des dispenses aux femmes qu'ils corrompent, 
si, une fois enceintes, elles se font avorter (Anonymous 16-17). William Fennor rapporte l'anecdote d'une jeune femme qui, sur le point d'être mariée, s'avère être enceinte d'un jésuite: "her belly [was] full of young bones, which [she] afterwards confessed were of the Jesuit's making ». L'auteur exhorte ses lecteurs à se défier de la familiarité de leurs épouses avec les jésuites qui auront tôt fait de les supplanter dans la chambre conjugale: «they themselves will vouchsafe to enter their closets » (Fennor 17-18).

C'est ainsi que la traditionnelle accusation anticléricale de sodomie est remplacée à cette époque en Angleterre par celle d'adultère (Dolan 89-93). Lors de son procès pour non-conformisme, Margaret Clitherow (c. 1556-86), est accusée d'avoir eu des relations sexuelles avec les prêtres qu'elle protégeait (Dillon 277). Un jeune élève de son école dit avoir été témoin de scènes qui ne laissent pas de doute à ce sujet; le conseiller Hurleston, siégeant au procès, s'en prend à celle qui, selon lui, a souillé le lit conjugal et déshonoré son mari : «It is not for religion that thou harbourest priests, but for harlotry » (Morris 414). Cette harangue exprime le sentiment populaire envers les missionnaires, qui envahissent l'intimité des époux et violent les liens les plus sacrés du foyer. À travers le scandale sexuel, si sensationnel, c'est le renversement des valeurs familiales que l'on craint, et pas seulement chez les protestants: en effet, les relations des récusantes et de leurs prêtres sont parfois l'objet de ressentiment, même chez les catholiques. Ainsi, Mr. Fermor porte-t-il plainte contre le clerc qui demeure sous son toit. Ce dernier, un certain Fr. Ashton, avait incité Mrs Fermor à remplacer les serviteurs du foyer par des domestiques de son choix et à renvoyer trois prêtres qui lui déplaisaient, sans que Mr. Fermor fût consulté sur ces changements. Lorsqu'il en prend connaissance, éclate une violente dispute au cours de laquelle il reproche au Fr. Ashton de s'être octroyé le rôle du paterfamilias. C'est alors, écrit-il, qu'il comprend que le missionnaire avait séduit sa femme pour mieux dominer sa famille et soumettre enfin l'époux, de gré ou de force, à son autorité : «Mr. Ashton hath told me diverse times if I would be ruled by him, I should have so good a wife as any man had in the country where I dwelt, insinuating what he could do with her » (Archives of the Archdiocese of Westminster 326).

Le devoir d'obéissance de l'épouse catholique est un problème difficile. En tant que femme, elle doit en principe obéissance à l'autorité patriarcale représentée par son époux; cependant, en tant 
que catholique, son allégeance ultime est due au clergé. Que faire si les ordres de l'un et de l'autre sont contradictoires? Doit-elle se soumettre à l'autorité civile ou religieuse ? Si pour les récusantes, et les catholiques en général, ce problème est parfois douloureux, les missionnaires sont très clairs. Le Concile de Trente (1545-63), en réaffirmant la primauté du clergé sur les laïcs, a libéré les femmes de leur devoir de soumission conjugale en matière de religion. Garnet écrit donc: "Your husbands over your souls have no authority and over your bodies but a limited power: but your heavenly spouse can condemn both unto everlasting fire, or reward with everlasting bliss » (Garnet 145). Pour la catholique, de telles injonctions impliquent donc un conflit inévitable entre devoirs maritaux et religieux.

Un certain antagonisme conjugal devient alors inévitable pour la récusante dont l'époux, lui, est membre de l'Église établie. Le chef de famille est en effet considéré comme responsable des infractions commises par tous les membres de son foyer. L'insubordination de sa femme a donc des conséquences souvent désastreuses sur les finances du paterfamilias; en outre, elle remet en cause sa capacité à diriger sa famille et le couvre de ridicule dans son voisinage. Le cas le plus connu de rébellion récusante contre l'autorité d'un époux conformiste est sans doute celui de Margaret Clitherow, à la tête de plusieurs centres catholiques dans la ville de York. C'est avec un respect non dissimulé que son biographe raconte comment elle rendait visite à son confesseur dès que ses devoirs d'épouse et de mère lui en laissaient le loisir (Mush 390). Quand elle demande si elle peut recevoir des prêtres sans l'accord de son mari, le prêtre, comme Garnet, l'assure que l'obéissance conjugale ne peut en aucun cas avoir précédence sur l'obéissance envers Dieu: « in this, your necessary duty to God, you are not any whit inferior to him » (392). La jeune femme continue ainsi ses activités, tandis que John Clitherow est la risée du voisinage et la supplie, si elle doit persister, d'être au moins plus discrète. Ainsi, il semble bien que le catholicisme des femmes justifie leur désobéissance domestique.

À travers de telles anecdotes se construit la représentation du missionnaire comme ennemi de l'ordre domestique, exhortant l'épouse à bafouer l'autorité légitime de son mari. Pour les protestants, ceci illustre parfaitement l'inversion catholique de l'ordre naturel. Pour Gee, les prêtres ont su jouer de la clandestinité de leur mission en Angleterre, tirant grand avantage de la proximité peu commune qu'elle leur offre 
avec les pénitentes qui les abritent et les protègent. Grâce à cette situation, ils développent leur ascendant, et exercent une influence qui, en aucun cas, ne saurait être convenable pour des serviteurs : «Thus, while they pretend that they are forced to creep into private houses for fear of persecution, they carry more dominion over the Family, than any Parish-Priest does in those Countries where Popish Religion publicly prevails» (Foot out of the Snare 80-1). Ayant renversé le rapport d'autorité entre maîtres et serviteurs, ils s'appliquent chaque jour à affaiblir l'infrastructure des rapports sociaux. Ils encouragent la destruction de tout devoir d'obéissance, d'abord entre conjoints, mais aussi entre parents et enfants; ils exhortent les nouveaux convertis à désavouer leur famille si celle-ci persiste dans l'hérésie. Le polémiste Thomas Morton s'insurge contre la dangereuse inversion de l'ordre domestique qu'est, selon lui, le catholicisme :

Wives are not bound to render due benevolence unto their husbands, if heretics. [...] For by the heresy of the father, the child is freed from obedience. [...] Heretics may not be termed either children or kindred; but according to the old law, Thy hand must be against them to spill their blood [...]. Shall we call this Religion, which [...] by no acknowledgement of Natural Duties of Wedlock, natural Parents, natural Children, natural Country, doth bowel up Nature, as it were, and deprive men of Humanity itself? (3-4).

Car c'est bien là la véritable peur qui nourrit le débat: le catholicisme dissout tout lien de loyauté et appelle les sujets à renverser l'autorité patriarcale.

Morton, parmi tant d'autres, concourt plus encore à la diabolisation des missionnaires en les désignant comme les ennemis naturels du bien commun: " Those snakes as do naturally sting as soon as they get warmth, may not be harboured in the bosom of the Commonwealth: but all popish priests profess rebellions as soon as they can presume of their strength » (13). Selon lui, en défiant leur époux, les insoumises comme Margaret Clitherow ne font rien moins que participer au renversement de la hiérarchie établie. Comme lui, de nombreux auteurs de l'ère moderne lient ainsi le microcosme familial aux enjeux nationaux (Anti-Papist 30). Parce qu'il promeut l'inversion des valeurs, le missionnaire menace le tissu social et l'intégrité politique de la nation. Bell avertit donc ses lecteurs :

Note here, gentle reader, that to depend upon the Jesuits is to depend upon the devil ; and consequently, that to follow the Jesuits and their 
bloody, tragic, and traitorous designs, is nothing else indeed, but to forsake God; to abandon his true fear and worship ; to be traitors to your prince; to be enemies to your native country. (Popish Tyrannie 8).

Au cours de l'époque moderne, et de plus en plus, le catholicisme est perçu comme l'antithèse de ce qui est cher au royaume; c'est l'Autre par excellence, une image inversée comme dans un miroir, entièrement irréconciliable avec l'anglicité. Si la doctrine éclairée de l'Anglicana Ecclesia est la voie du salut, la «superstition » romaine ne peut être que le chemin de la perdition. Le «papiste»s'apparente ainsi au serpent miltonien qui, dans Paradise Lost, devine la fragilité d'Ève et se réjouit de sa nature imparfaite. Il la séduit pour la convaincre d'agir selon son gré, mais le but ultime de sa séduction n'est autre que la corruption de l'humanité et le renversement de l'ordre divin. La séduction de la gent féminine est le moyen le plus sûr de réaliser ses desseins. C'est ainsi que, tandis que l'Angleterre moderne construit son identité anglicane et nationale dans l'altérité avec l'Église de Rome, elle donne à la récusante une importance qu'elle n'avait probablement pas pour ses coreligionnaires; car dans la construction de cet anticatholicisme manichéen, le rôle des femmes, exagéré, est nécessaire à la représentation du monde catholique comme un monde inversé. La polémique insiste donc sur le fait que c'est en envoûtant les femmes et en les détournant du droit chemin que les missionnaires parviennent à parasiter la société anglaise. C'est cette séduction spirituelle, pétrie de mensonge et de manipulation, qui permet à ces agents du mal de corrompre les liens de la famille et de la société tout entière, pour finalement atteindre leur but ultime et renverser l'ordre établi.

Laurence LUX - STERRITT

Université d'Aix-Marseille I. 


\section{OUVRAGES CITÉS}

\section{SOURCES PRIMAIRES}

ANONYMOUS. The Black Box of Roome Opened. From Whence are Revealed the Damnable Bloody Plots, practices and Behaviour of Iesuites, Priests, Papists, and Other Recusants in Generall. London: s. n., 1641.

ANSTRUTHER, Godfrey. Vaux of Harrowden, a Recusant Family. Newport : R. H. Johns, 1953.

ANTI-PAPIST. A Fair Warning to take heed of Popery, or a Short and True History of the Jesuits Fiery Practices and Powder-Plots, to destroy Kings, ruin Kingdoms and lay Cities Waste. London: Thomas Parkhurst, 1679.

ArCHIDIOCÈSE DE WESTMINSTER. Archives. Vol. 37, $\mathrm{n}^{\circ} 97$.

BAXTER, John. A Toile for Two-Legged Foxes. London: Thomas Man, 1600.

BECON, Thomas. The Displaying of the Popish Masse, wherein Thou Shalt See what a Wicked Idoll the Masse is, and What Great Difference there is Between the Lords Supper and the Popes Masse. London : Company of Stationers, 1637.

BELL, Thomas. The Anatomie of Popish Tyrannie. London: Richard Bankworth, 1603.

- The Downfall of Poperie, Proposed by Way of a New Challenge to all the Iesuits and Iesuited or Italianized Papists. London: Arthur Johnson, 1604.

- The Hunting of the Romish Foxe. London: Richard Bradocke, 1598.

BERNARD, Richard. A Key of Knowledge for the Opening of the Secret Mysteries of St Johns Mysticall Revelation. London : Felix Kyngston, 1617.

CARAMAN, Philip, ed. Henry Garnet 1555-1606 and the Gunpowder Plot. London : Longmans, 1964.

—. John Gerard. The Autobiography of an Elizabethan. London: Longmans, 1956.

COMPTON, Henry. The Jesuites' Intrigues, with the Private Instructions of that Society to their Emissaries. London: Benjamin Tooke, 1669. 
FenNor, William. Pluto his Travailes, or the Deuils Pilgrimage to the Colledge of Iesuits. London : Joseph Hunt, 1612.

GARNET, Henry. A Treatise of Christian Renunciation. Ed. D. M. Rogers. English Recusant Literature, 1558-1640. Vol. 47. Menston: Scholar Press, 1970.

GEE, John. New Shreds of the Old Snare. London: Robert Milbourne, 1624.

- The Foot out of the Snare. With a Detection of Sundry Late Practices and Impostures of the Priests and Iesuites In England. London: Robert Milbourne, 1624.

HarsnetT, Samuel. A Declaration of Egregious Popish Impostures to With-draw the Harts of her Maiesties Subjects from their Allegeance and from the Truth of Christian Religion Professed in England, under the Pretence of Casting out Devils. London: James Roberts, 1603.

Milton, John. Areopagitica. A Speech for the Liberty of Unlicensed Printing to the Parliament of England. 1644. John Milton, The Major Works. Ed. Stephen Orgel and Jonathan Goldberg. Oxford : Oxford UP, 2003.

MORTON, Thomas. An Exact Account of Romish Doctrine in the Case of Conspiracies and Rebellion. London: John Starkey, 1679.

MusH, John. The Life and Death of Mistress Margaret Clitherow. 1586. The Troubles of Our Catholic Forefathers. Vol. 3. Ed. John Morris. London : Burns and Oates, 1877.

\section{SOURCES SECONDAIRES}

ANSTRUTHER, Godfrey. Vaux of Harrowden, a Recusant Family. Newport: R. H. Johns, 1953.

Bossy, John. The English Catholic Community, 1570-1850. London: Darton, Longman and Todd, 1976.

DiLlon, Anne. Martyrdom in the English Catholic Community, 15351603. Aldershot: Ashgate, 2002.

DOLAN, Frances. Whores of Babylon. Catholicism, Gender and Seventeenth Century Print Culture. Ithaca : Cornell UP, 1999.

LAKE, Peter. "Anti-Popery: the Structure of a Prejudice». Conflict in Early Stuart England. Ed. Richard Cust and Ann Hughes. London: Longman, 1989. 72-106. 
- - and Michael QUESTIER. The Antichrist's Lewd Hat: Protestants, Papists and Players in Post-Reformation England. New Haven: Yale UP, 2002.

MAROTTI, Arthur. Religious Ideology and Cultural Fantasy. Catholic and Anti-Catholic Discourses in Early Modern England. Notre Dame : U of Notre Dame P, 2005.

MILTON, Anthony. «A Qualified Intolerance: The Limits and Ambiguities of Early Stuart Anti-Catholicism». Catholicism and Anti-Catholicism in Early Modern English Texts. Ed. Arthur Marotti. Basingstoke: Macmillan, 1999. 63-84.

ROWLANDS, Marie. «Recusant Women 1560-1640». Women in English Society 1500-1800. Ed. Mary Prior. London: Methuen, 1985. 149-80.

SEGUIN, Colleen. "'Ambiguous Liaisons' : CatholicWomen's Relationships with their Confessors in Early Modern England». Archiv für Reformationsgeschichte 95 (2004) : 156-85.

SHELl, Alison. Catholicism, Controversy and the English Literary Imagination, 158-1660. Cambridge: Cambridge UP, 2006.

TuMBLESON, Raymond. Catholicism in the English Protestant Imagination: Nationalism, Religion, and Literature, 1600-1745. Cambridge : Cambridge UP, 1998.

Walsham, Alexandra. Church Papists : Catholicism, Conformity and Confessional Polemic in Early Modern England. Woodbridge: Boydell, 1993.

YATES, Julian. «Parasitic Geographies: Manifesting Catholic Identity in Early Modern England ». Catholicism and Anti-Catholicism in Early Modern English Texts. Ed. Arthur Marotti. Basingstoke: Macmillan, 1999. 85-115. 